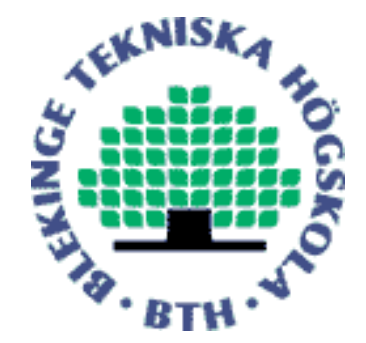

Copyright (C) 2009 IEEE.

Citation for the published paper:

On Confidence and Response Times of Human Observers in Subjective Image Quality Assessment

Ulrich Engelke, Anthony Maeder, Hans-Jürgen Zepernick

International Conference on Multimedia and Expo

2009 New York City

This material is posted here with permission of the IEEE. Such permission of the IEEE does not in any way imply IEEE endorsement of any of BTH's products or services Internal or personal use of this material is permitted. However, permission to reprint/republish this material for advertising or promotional purposes or for creating new collective works for resale or redistribution must be obtained from the IEEE by sending a blank email message to pubs-permissions@iee.org.

By choosing to view this document, you agree to all provisions of the copyright laws protecting it. 


\title{
ON CONFIDENCE AND RESPONSE TIMES OF HUMAN OBSERVERS IN SUBJECTIVE IMAGE QUALITY ASSESSMENT
}

\author{
Ulrich Engelke ${ }^{\dagger}$, Anthony Maeder ${ }^{*}$, and Hans-Jürgen Zepernick ${ }^{\dagger}$ \\ ${ }^{\dagger}$ Blekinge Institute of Technology, PO Box 520, 37225 Ronneby, Sweden, E-mail: uen@bth.se \\ *University of Western Sydney, Locked Bag 1797, Penrith South DC, NSW 1797, Australia
}

\begin{abstract}
Mean opinion scores obtained in subjective image quality experiments are widely accepted as measures of perceived visual quality. They have, however, a strong limitation regarding the reliability of the rated quality, since there is no explicit information as to whether the human observer experienced difficulties when judging image quality. We thus suggest that additional information about the observers confidence should be provided along with the actual quality measure. In this paper, we analyse two ways of obtaining this confidence measure; firstly as a confidence score given by the human observer and secondly as an indirect measure of the observers response time to provide the quality score. We reveal strong relationships of confidence scores and response times to the quality scores. We further propose a model to predict observer confidence based on the quality scores and response times.
\end{abstract}

Index Terms - Subjective image quality experiment, observer confidence, observer response times.

\section{INTRODUCTION}

Mean opinion scores (MOS) obtained in subjective quality experiments are to date the only widely recognised measures of perceived visual quality [1]. The drawback of subjective experiments, however, is that they are usually time consuming and expensive. Also, MOS typically cannot be obtained in real-time, thus essentially limiting the application of subjective experiments for in-service monitoring of visual quality. On the other hand, MOS are typically used as a ground truth to design objective quality metrics which in turn can be used to automatically predict subjective quality [2].

Rating the quality of images may not necessarily be an easy task for a human observer, in particular when there is a variety of distortions apparent in the visual content. In order to obtain a measure of reliability of a particular MOS, confidence intervals (CI) are usually computed to quantify the disagreement between participants. However, CI do not directly capture the confidence with which a particular observer rated an image. There may be, for instance, artifacts that are easy to rate but for which the opinions of the participants are widely spread. Furthermore, artifacts may not even be perceived by every participant due to masking effects and consequently, the CI could be wide even though many observers did their quality rating with high confidence.

Given the above, we analyse two different ways to provide reliability information in addition to the CI. The first is related to the confidence of a human observer when rating the quality of an image, obtained by a confidence score provided by the observer. In some cases it may be inconvenient though to require too much information from a participant during a subjective experiment. Therefore, we consider another measure which we believe is related to the confidence of the observer; the response time which the human observer requires to give a quality rating. Given the above we hypothesize that:

H1. It is easier to rate an image if its quality is either very good or very bad while images of medium quality are harder to judge. As a measure of difficulty when judging image quality we consider a confidence score given by a human observer.

H2. The confidence of a human observer when rating the quality of an image is strongly related to the response time of the quality rating. As such, we expect a longer response time for images that are harder to judge.

H3. Observer confidence can be predicted with reasonable accuracy based on the given quality score in combination with the response time measured. Such a confidence prediction may be used as a measure of reliability of a particular MOS.

The aims of this paper are twofold. Firstly, we aim to establish relationships between quality scores, confidence scores, and response times obtained from a subjective image quality experiment. Secondly, we aim to model the prediction of mean confidence scores using the quality scores and response times. The predicted mean confidence scores may then serve as a non-intrusive measure of observer confidence.

The paper is organised as follows. Section 2 describes the subjective image quality experiment. Section 3 analyses the relationships between the quality scores, confidence scores, and response times as they were obtained during the experiment. Section 4 discusses the prediction of mean confidence scores. Finally, conclusions are drawn in Section 5. 


QUALITY SCORE
\begin{tabular}{|c|c|}
\hline Very Good & 5 \\
\hline Good & 4 \\
\hline Fair & 3 \\
\hline Bad & 2 \\
\hline Very Bad & 1 \\
\hline
\end{tabular}

CONFIDENCE SCORE
\begin{tabular}{|c|c|}
\hline Very High & 5 \\
\hline High & 4 \\
\hline Medium & 3 \\
\hline Low & 2 \\
\hline Very Low & 1 \\
\hline
\end{tabular}

Fig. 1. Scales for quality scores and confidence scores.

\section{SUBJECTIVE IMAGE QUALITY EXPERIMENT}

We conducted a subjective image quality experiment at the School of Computing and Mathematics at the University of Western Sydney. The experiment procedures were designed according to ITU-R Rec. BT.500-11 [3]. A total of 15 people participated in the experiment, of which 5 were female and 10 were male. The average age of all participants was 42 years.

The participants were presented a number of grey scale images encoded in Joint Photographic Experts Group (JPEG) format. Seven widely adopted reference images (Barbara, Elaine, Goldhill, Lena, Mandrill, Pepper, Tiffany) were used to create a set of 80 test images. For this purpose a simulation model of a wireless channel was utilised to induce a number of different artifacts in the test images, including blocking, blur, ringing, intensity masking, and combinations thereof. The test set covered a wide range of severities from almost invisible artifacts to highly distorted images. The experiment was divided into two sessions of about 10 minutes duration each. In each session, 40 test images were shown along with the 7 reference images. Each image was presented for $8 \mathrm{sec}-$ onds with a grey screen shown in between for 5 seconds.

During the grey screen in between the images, the participants were asked to rate the image quality on a 5-point scale, with 5 being highest quality. In order for the participants to have an idea about the range of artifacts that could be expected during the experiment, a set of 7 training images was shown prior to the actual test images. The training images covered a wide range of artifact severities. In addition to the quality scores (QS) the participants were asked to provide a confidence score (CS) on a 5-point scale, as a measure of how difficult is was to judge the quality of a particular image. The higher the confidence, the easier it was to rate the quality. Both the quality scale and the confidence scale, as used in the experiment, are shown in Fig. 1. Finally, the response times (RT) that the participant took to provide both the QS and the CS have been recorded by the experimenter.

\section{ANALYSIS}

In this section we analyse the relationship between the QS, CS, and RT. For this purpose, we define the means over all participants for each of the images. In particular, the mean for the quality scores, represented by the MOS, is denoted as $\mu_{Q S}$, the mean confidence scores (MCS) are denoted as $\mu_{C S}$, and the mean response times (MRT) are denoted as $\mu_{R T}$.

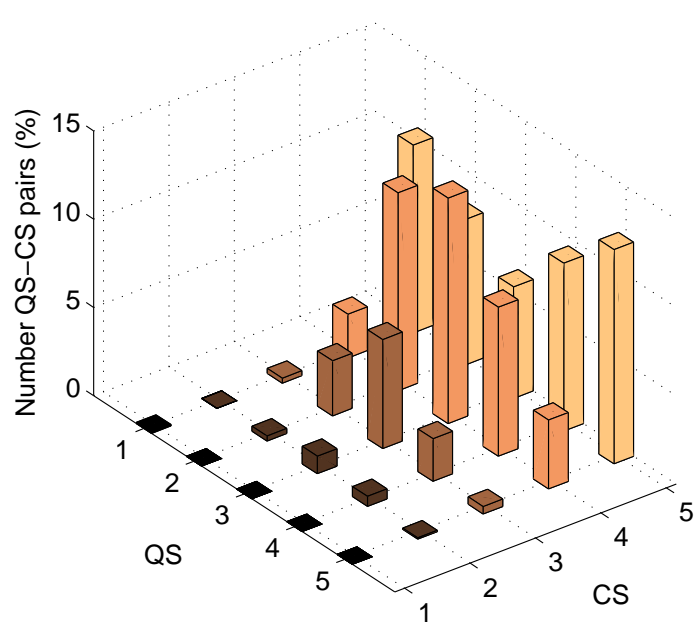

Fig. 2. Number of occurrences of pairs of QS and CS.

Table 1. Percentage of QS and CS.

\begin{tabular}{c||c|c|c|c|c}
\hline & 1 & 2 & 3 & 4 & 5 \\
\hline \hline QS & $13.48 \%$ & $22.84 \%$ & $26.24 \%$ & $20.92 \%$ & $16.52 \%$ \\
\hline CS & $0.07 \%$ & $1.91 \%$ & $12.41 \%$ & $38.79 \%$ & $46.81 \%$ \\
\hline
\end{tabular}

\subsection{Occurrence of pairs of QS and CS}

We hypothesized that it may be easier for a human observer to judge the quality of images at either end of the quality scale and that it may be harder to judge quality in the middle range of qualities (see H1). As such, one would expect high CS at either end of the quality scale. This hypothesis is confirmed by the analysis of the QS and CS obtained in the subjective experiment. The number of particular combinations of QS and CS as given by the participants are shown in Fig. 2. One can see that for QS at both the high end of the scale $(\mathrm{QS}=5)$ and the low end of the scale (QS =1), the confidence of the majority of human observers has been very high. This very high confidence drops towards the middle of the quality scale. However, one can see that the lower values of CS $(\leq 4)$ are predominant in the middle of the quality scale.

It is also interesting to note that the whole spectrum of QS has been covered by the participants. However, there is a strong tendency towards higher values in case of the CS. The exact percentages of particular QS and CS, as compared to the total number of the respective scores, are provided in Table 1.

\subsection{Average RT for QS and CS}

We further hypothesized that RT may be longer for images that are harder to judge since the participant might require more time to make a decision (see H2). This may in turn be inversely related to the CS, meaning, a higher confidence should result in a quicker response. Thus RT may provide an indirect measure of observer confidence.

The average RT over all participants and all images are 


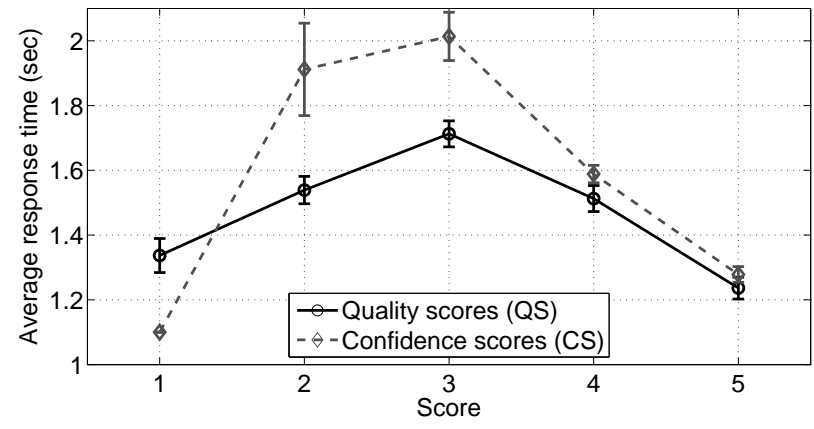

Fig. 3. Average RT (with standard error of the mean) over all participants and images relating to particular QS and CS.

shown in Fig. 3 for both CS and QS. One can see that the RT generally increases with decreasing CS. However, the drop of RT for CS = 1 seems contradictory, as one would expect a longer RT for a lower CS. It should be noted here that there was only one single $\mathrm{CS}=1$, as can be observed from the negligible percentage in Table 1. As such, this value does not have statistical significance and may in fact constitute an outlier.

From Fig. 3 one can also observe that the RT are increasing towards the middle of the quality scale, which is in alignment with the decreasing CS toward the middle of the quality scale (see Fig. 2). This indicates that RT may also contribute information about the reliability of MOS.

\subsection{Correlations between QS, CS, and RT}

The above findings indicate that there is a strong relationship between QS, CS, and RT. In fact, CS and RT are not directly related to QS but rather to the distance of QS to the middle of the quality scale $m_{Q S}=3$. Therefore, we define a delta-QS (DQS) measure as follows

$$
\mu_{Q S}^{\Delta}=\left|\mu_{Q S}-m_{Q S}\right|
$$

In this respect, $\mu_{Q S}^{\Delta}$ is thought to be related to $\mu_{C S}$ and $\mu_{R T}$ since the QS at either end of the quality scale have been shown in the previous sections to result in a higher CS and a lower RT. To further quantify the interdependencies between QS, CS, and RT we consider the Pearson linear correlation coefficient given by

$$
\rho_{P}(u, v)=\frac{\sum_{k=1}^{K}\left(u_{k}-\bar{u}\right)\left(v_{k}-\bar{v}\right)}{\sqrt{\sum_{k=1}^{K}\left(u_{k}-\bar{u}\right)^{2}} \sqrt{\sum_{k=1}^{K}\left(v_{k}-\bar{v}\right)^{2}}}
$$

where $u_{k}$ and $v_{k}$ represent any combination of $\mu_{Q S}^{\Delta}, \mu_{C S}$, and $\mu_{R T}$ and $\bar{u}$ and $\bar{v}$ are the means of the respective data sets over all images. As such, $\rho_{P}$ quantifies the linear dependence between the two data sets and thus, the accuracy with which one data set can be represented by another.
Table 2. Prediction function parameters.

\begin{tabular}{l||c|c|c}
\hline & $a$ & $b$ & $c$ \\
\hline \hline Linear fit (4) & 3.802 & 0.483 & - \\
\hline Power fit (5) & 2.679 & 2.236 & -0.829 \\
\hline
\end{tabular}

We have computed the correlation coefficient $\rho_{P}$ for all three combinations of $\mu_{Q S}^{\Delta}, \mu_{C S}$, and $\mu_{R T}$, to establish a full overview of the interdependencies. The correlations are given as follows

$$
\begin{aligned}
& \rho_{P}\left(\mu_{Q S}^{\Delta}, \mu_{C S}\right)=0.825 \\
& \rho_{P}\left(\mu_{Q S}^{\Delta}, \mu_{R T}\right)=-0.714 \\
& \rho_{P}\left(\mu_{C S}, \mu_{R T}\right)=-0.696
\end{aligned}
$$

It can be seen from the correlations that there is indeed a strong relationship between all three measures. In particular, DQS and MCS observe a very distinct correlation. The negative correlation coefficients indicate that MRT is inversely related to both DQS and MCS.

\section{PREDICTION OF OBSERVER CONFIDENCE}

From the analysis in the previous sections it is apparent that MCS is strongly related to both DQS and MRT. Even though both DQS and MRT already provide a reasonable indication of an observers confidence when rating image quality, one may suspect that a combination of DQS and MRT could result in a further improvement of confidence prediction (see $\mathrm{H} 3$ ). In this section we thus aim on modelling the prediction of observer confidence based on DQS and MRT. In this respect we first establish prediction functions for both DQS and MRT and then apply a combinatorial model to predict MCS.

\subsection{Prediction of MCS from either DQS or MRT}

Prediction functions have been established independently for DQS and MRT using linear and non-linear regression, respectively. The fittings are shown in Fig. 4 and Fig. 5. In the case of DQS a linear mapping is given as

$$
\mu_{C S}^{(Q S)}(a, b)=a+b \cdot \mu_{Q S}^{\Delta}
$$

On the other hand, for MRT we have obtained a non-linear relationship in terms of a power function as

$$
\mu_{C S}^{(R T)}(a, b, c)=a+b \cdot \mu_{R T}^{c}
$$

This non-linear relationship is also apparent in the lower linear correlation coefficient $\rho_{P}\left(\mu_{C S}, \mu_{R T}\right)$ as compared to the correlation coefficient $\rho_{P}\left(\mu_{Q S}^{\Delta}, \mu_{C S}\right)$ (see (3)). The parameters for both prediction functions are summarised in Table 2. 


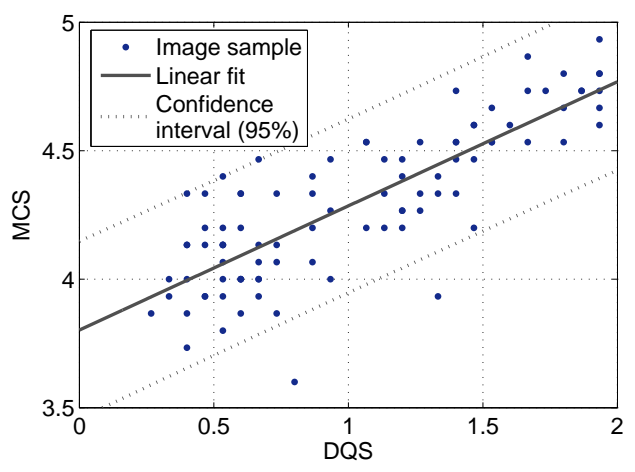

Fig. 4. Linear fit between DQS and MCS.

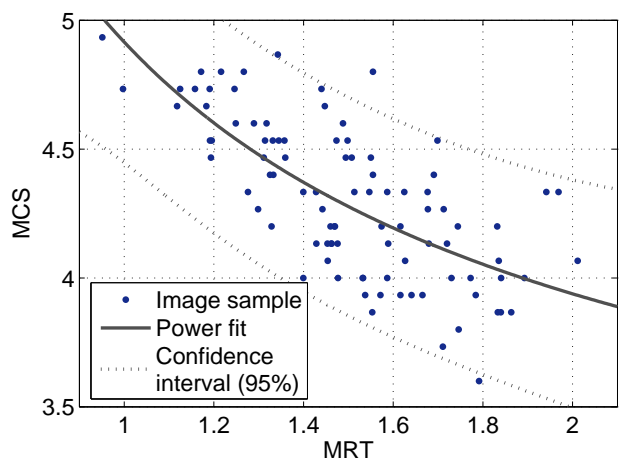

Fig. 5. Power fit between MRT and MCS.

\subsection{Combinatorial prediction model}

The $L_{p}$-norm, also known as Minkowski metric, is widely deployed as a combinatorial metric [4]. In our case, we use a slight modification, the weighted $L_{p}$-norm, which additionally assigns relevance weights to each of the combined data sets. Given the prediction functions $\mu_{C S}^{(Q S)}$ and $\mu_{C S}^{(R T)}$ in (4) and (5), respectively, we define a combinatorial model to predict MCS as follows

$$
\mu_{C S}^{p r e d}(\omega, p)=\left[\omega \cdot\left(\mu_{C S}^{(Q S)}\right)^{p}+(1-\omega) \cdot\left(\mu_{C S}^{(R T)}\right)^{p}\right]^{\frac{1}{p}}
$$

where $p \in \mathbb{Z}^{+}$is the Minkowski parameter and $\omega \in[0,1]$ is the relevance weight. Optimal parameters $p_{O p t}$ and $\omega_{O p t}$ are then obtained by exhaustive search in the parameter space. Given the model in (6) we obtained a correlation coefficient of $\rho_{P}\left(\mu_{C S}, \mu_{C S}^{p r e d}\right)=0.843$, thus, improving the prediction performance as compared to using MOS or MRT independently. However, we found that a simple model given by

$$
\mu_{C S}^{p r e d}(\omega, p)=\left[\omega \cdot \mu_{Q S}^{p}+(1-\omega) \cdot\left(\frac{1}{\mu_{R T}}\right)^{p}\right]^{\frac{1}{p}}
$$

can provide an even slightly better prediction performance of $\rho_{P}\left(\mu_{C S}, \mu_{C S}^{\text {pred }}\right)=0.845$ while at the same time simplifying model complexity. Therefore, we propose the model in (7) for

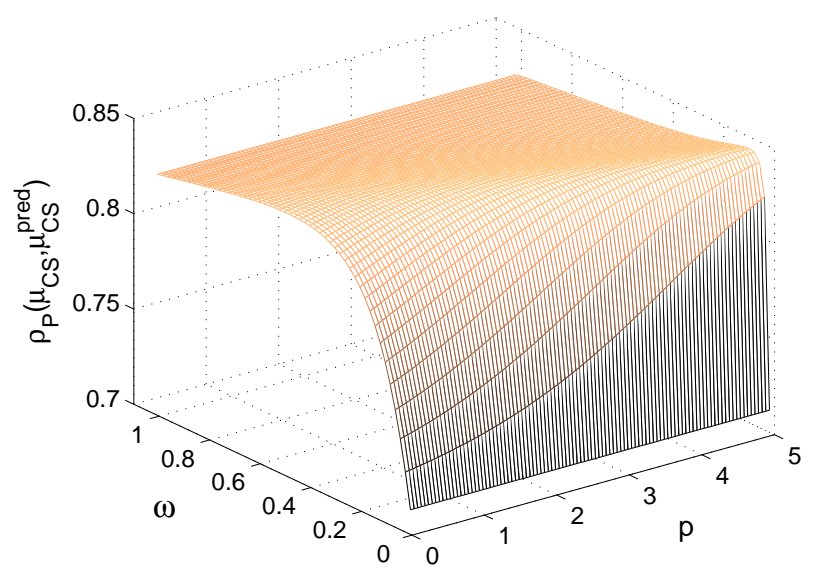

Fig. 6. Correlations $\rho_{P}\left(\mu_{C S}, \mu_{C S}^{\text {pred }}\right)$ for $\omega$ and $p$.

prediction of observer confidence. The optimal parameters for this model were obtained as

$$
p_{O p t}=3.036, \quad \omega_{O p t}=0.184
$$

The dependance of the proposed model on $p$ and $\omega$ is shown in Fig. 6 in terms of the correlation coefficient $\rho_{P}\left(\mu_{C S}, \mu_{C S}^{\text {pred }}\right)$. One can see that the model is highly dependent on the relevance weight $\omega$ but less on the Minkowski parameter $p$.

\section{CONCLUSIONS}

In this paper we analysed the relationship between QS, CS, and RT as obtained in our subjective image quality experiment. We have shown that valuable information about an observers confidence when rating image quality can be derived from the actual QS and also the RT. We further proposed a model to predict MCS with reasonable accuracy from a combination of MOS and MRT. In future work, we will analyse the relationship of our prediction model with CI.

\section{REFERENCES}

[1] S. Winkler, Digital Video Quality - Vision Models and Metrics, John Wiley \& Sons, 2005.

[2] U. Engelke and H. J. Zepernick, "Pareto optimal weighting of structural impairments for wireless imaging quality assessment," in Proc. of IEEE Int. Conf. on Image Processing, Oct. 2008, pp. 373-376.

[3] International Telecommunication Union, "Methodology for the subjective assessment of the quality of television pictures,” Rec. BT.500-11, ITU-R, 2002.

[4] H. de Ridder, "Minkowski-metrics as a combination rule for digital-image-coding impairments," in Proc. of IS\&T/SPIE Human Vision, Visual Processing, and Digital Display III, Jan. 1992, vol. 1666, pp. 16-26. 